\title{
"The Incubation Period for Void Swelling and its Dependence on Temperature, Dose Rate, and Dislocation Structure Evolution"
}

\author{
M. P. Surh, J. B. Sturgeon, W. G. Wolfer
}

Lawrence

Livermore

National

Laboratory

This article was submitted to

$21^{\text {st }}$ Symposium on Effects of Radiation on Materials, Tucson, AZ, June 18-20, 2002

U.S. Department of Energy

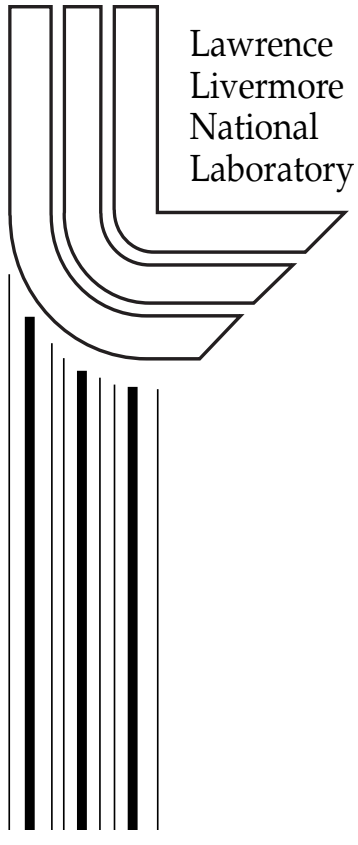

June 13, 2002 


\section{DISCLAIMER}

This document was prepared as an account of work sponsored by an agency of the United States Government. Neither the United States Government nor the University of California nor any of their employees, makes any warranty, express or implied, or assumes any legal liability or responsibility for the accuracy, completeness, or usefulness of any information, apparatus, product, or process disclosed, or represents that its use would not infringe privately owned rights. Reference herein to any specific commercial product, process, or service by trade name, trademark, manufacturer, or otherwise, does not necessarily constitute or imply its endorsement, recommendation, or favoring by the United States Government or the University of California. The views and opinions of authors expressed herein do not necessarily state or reflect those of the United States Government or the University of California, and shall not be used for advertising or product endorsement purposes.

This is a preprint of a paper intended for publication in a journal or proceedings. Since changes may be made before publication, this preprint is made available with the understanding that it will not be cited or reproduced without the permission of the author.

This work was performed under the auspices of the United States Department of Energy by the University of California, Lawrence Livermore National Laboratory under contract No. W-7405-Eng-48.

This report has been reproduced directly from the best available copy.

Available electronically at http://www.doc.gov/bridge

Available for a processing fee to U.S. Department of Energy

And its contractors in paper from

U.S. Department of Energy

Office of Scientific and Technical Information

P.O. Box 62

Oak Ridge, TN 37831-0062

Telephone: (865) 576-8401

Facsimile: (865) 576-5728

E-mail: reports@adonis.osti.gov

Available for the sale to the public from

U.S. Department of Commerce

National Technical Information Service

5285 Port Royal Road

Springfield, VA 22161

Telephone: (800) 553-6847

Facsimile: (703) 605-6900

E-mail: orders@ntis.fedworld.gov

Online ordering: http://www.ntis.gov/ordering.htm

OR

Lawrence Livermore National Laboratory

Technical Information Department's Digital Library

http://www.llnl.gov/tid/Library.html 
Michael P. Surh ${ }^{1}$, J.B. Sturgeon ${ }^{1}$, and W. G. Wolfer ${ }^{1}$

"The Incubation Period for Void Swelling and its Dependence on Temperature, Dose Rate, and Dislocation Structure Evolution"

Reference: Surh, M.P., Sturgeon, J.B., and Wolfer, W.G., "The Incubation Period for Void Swelling and its Dependence on Temperature, Dose Rate, and Dislocation Structure Evolution", Effects of Radiation on Materials, ASTM STP 1447, M.L. Grossbeck, Ed., ASTM International, West Conshohocken, PA, 2003.

\begin{abstract}
Void swelling in structural materials used for nuclear reactors is characterized by an incubation period whose duration largely determines the usefulness of the material for core components. Significant evolution of the dislocation and void microstructures that control radiation-induced swelling can occur during this period. Thus, a theory of incubation must treat time-dependent void nucleation in combination with dislocation evolution, in which the sink strengths of voids and dislocations change in concert. We present theoretical results for void nucleation and growth including the time-dependent, self-consistent coupling of point defect concentrations to the evolution of both void populations and dislocation density. Simulations show that the incubation radiation dose is a strong function of the starting dislocation density and of the dislocation bias factors for vacancy and interstitial absorption. Irradiation dose rate and temperature also affect the duration of incubation. The results are in general agreement with experiment for high purity metals.
\end{abstract}

\title{
Introduction
}

Irradiation damage in metals leads to the formation of voids, with an associated volumetric swelling of the material. These vacancy defect clusters can also pin dislocations and so alter material strength and ductility. Through these changes, voiding may limit the utility of a given structural material in reactor environments. Void swelling may develop over very long times, so it is useful to understand, in advance, the parameters that control swelling behavior under irradiation. Recent experimental analysis reveals the importance of a transient stage (the so-called incubation period) on swelling measurements (Garner 1993). The duration of the incubation depends on environmental parameters, such as temperature and dose rate. The subsequent, steadystate swelling is largely independent of temperature and dose rate, instead being determined by the intrinsic material properties. Separating the two regimes of behavior can be difficult, if the experiment is not performed to sufficiently large total dose.

\footnotetext{
${ }^{1}$ Lawrence Livermore National Laboratory, 7000 East Ave., Livermore, CA 94550
} 
The steady-state swelling regime is reasonably well understood (Katz and Wiedersich 1971, Russell 1971, Brailsford and Bullough 1972, Garner 1984). It requires a net segregation of radiation-induced defects: interstitial atoms to climbing dislocations and vacancies to the growing voids. The segregation occurs due to thermal diffusion of defects, biased by stress-induced interactions between the mobile defects and the larger defect sinks/sources. In the absence of a driving force, the vacancies and interstitials will tend to be absorbed in equal numbers at voids and dislocations, such that no net swelling occurs. The existence of an incubation period may be attributed to changes in the material microstructure that are required for vacancy-interstitial segregation to proceed but that do not immediately lead to significant volume changes. Absent preexisting voids, the irradiation-induced defects would tend to be absorbed at the dislocations in equal numbers for vacancies and interstitials, so that no net swelling could occur. Thus, it is reasonable to expect that the nucleation of voids dictates the incubation period for radiation swelling.

Earlier simulations of void nucleation and growth with a time-independent dislocation density also support this interpretation (Surh, Sturgeon, and Wolfer). They show that void nucleation precedes the appearance of steady swelling. The temperature, dose rate, and initial dislocation density all influence the duration of this nucleation stage. For example, high initial dislocation densities in cold-worked material absorb excess vacancy monomers, thereby reducing the supersaturation and the rate of stable vacancy cluster nucleation. The resulting, prolonged period of void nucleation somewhat resembles the experimental incubation behavior. However, this model is known to be an oversimplification, since the dislocation density simultaneously evolves under irradiation. While the initial stages of irradiation occur in the absence of voids or swelling, the microstructure will still undergo changes due to variations in the local environment of the dislocation segments. A realistic treatment of the incubation process should include full co-evolution of the dislocation and void sub-systems.

We present here calculations that evaluate the full, time-dependent distribution of vacancy clusters and the dislocation density, all within a mean-field framework. The vacancy cluster distribution ranges from monomers to voids of arbitrary size. We use a modification of an earlier theory of void nucleation and growth (Wehner and Wolfer 1985). The stochastic, atomic processes of void growth or shrinkage are included by master equation and Fokker-Planck treatments of the void size distribution function. The size-dependent void bias factors are calculated according to (Sniegowski and Wolfer 1983). Void growth and fluctuation rates are determined from a self-consistent calculation of the thermal and radiation-induced vacancy and interstitial monomer populations (Wehner and Wolfer 1985). There is no production bias in this model; the radiation damage is introduced as vacancy and interstitial monomers. The dislocation subsystem is modeled entirely as network dislocations, in terms of a single density parameter. Interstitial aggregation is not allowed, as dislocation loops are not considered. Dislocation bias factors are calculated according to previously described methods (Sniegowski and Wolfer 1983). There is a cutoff distance used in the derivation that is related to the average density of dislocations. We make two choices for that parameter here. The first is to calculate dislocation bias factors assuming a density of $6 \times 10^{14} \mathrm{~m}^{-2}$, a 
typical terminal density under steady irradiation. This is the procedure in (Wehner and Wolfer 1985), where it would be expected give the experimentally observed asymptotic swelling rates for calculations with a fixed dislocation density. Our second choice is to calculate the bias factors using a cutoff obtained from the instantaneous value of the evolving dislocation density. That density is evolved according to a model (Wolfer and Glasgow 1985) that incorporates dislocation-dislocation annihilation processes along with a dislocation multiplication from pinned dislocations undergoing irradiation-driven, biased climb. The model includes one free parameter, namely, the mesh length or pinning density (set to a value of $400 \mathrm{~nm}$ to fit the observed terminal dislocation densities in irradiated steels).

We verify that our implementation of the dislocation evolution model reproduces earlier results for annealing in 316 stainless steel. We also check that the combined void plus dislocation simulations conserve mass, both with and without irradiation. The method is computationally efficient to temperatures of $650 \mathrm{C}$, at which point the stable void size becomes too large for efficient simulations. For all lower temperatures, our simulations extend to much longer times than previous treatments of stochastic void nucleation. We apply this method to a model of a high-purity type-316 austenitic stainless steel. The material parameters are taken from an earlier study of dislocation evolution under irradiation and are listed in Table I. We consider three different irradiation dose rates $\left(10^{-6}, 10^{-7}, 10^{-8} \mathrm{dpa} / \mathrm{s}\right)$ and six temperatures $(340 \mathrm{C}$ to $540 \mathrm{C}$ in $40 \mathrm{C}$ increments). The dislocation density is started at a value of $6 \times 10^{13} \mathrm{~m}^{-2}$, corresponding to a solution annealed limit.

Table 1. Model materials parameters for type-316 stainless steel.

\begin{tabular}{|l|l|}
\hline Cascade efficiency & 0.1 \\
\hline Lattice parameter, $a_{0}$ & $0.3639 \mathrm{~nm}$ \\
\hline Burgers vector & $0.2573 \mathrm{~nm}$ \\
\hline Shear Modulus & $82.95 \mathrm{Gpa}$ \\
\hline Poisson's Ratio & 0.264 \\
\hline Vacancy migration energy & $1.92 \times 10^{-19} \mathrm{~J}$ \\
\hline Vacancy formation energy & $2.88 \times 10^{-19} \mathrm{~J}$ \\
\hline Pre-exponential factor $D_{v}^{0}$ & $1.29 \times 10^{-6} \mathrm{~m}^{2} / \mathrm{s}$ \\
\hline Vacancy relaxation volume & $0.2 \Omega$ \\
\hline Vacancy shear polarizability & $-2.4 \times 10^{-18} \mathrm{~J}$ \\
\hline Interstitial shear polarizability & $-2.535 \times 10^{-18}, \mathrm{~J}$ \\
\hline
\end{tabular}

\section{Results}

It is easy to see a temperature-dependent incubation-like period in the simulated void swelling versus time. Volumetric swelling curves are presented in Fig. 1 for a series of temperatures $\left(340 \mathrm{C}\right.$ to $540 \mathrm{C}$ ) for an irradiation dose rate of $10^{-6} \mathrm{dpa} / \mathrm{s}$ and an initial dislocation density of $6 \times 10^{13} \mathrm{~m}^{-2}$. The data shown in Fig. 1 are obtained using a constant dislocation bias factor, as in (Wehner and Wolfer 1985). The values for 316 stainless steel are 1.63 for interstitials and 1.04 for vacancies. The simulations show a brief 
incubation period, which increases in duration at lower temperatures. Subsequently, the swelling rates at different temperatures are comparable, around $0.85 \% / \mathrm{dpa}$. This asymptotic rate is largely dictated by the dislocation bias factors. Overall, the predicted swelling behavior is similar to experiment, both in the appearance of an incubation-like feature and in terms of the numerical value of the asymptotic slope. However, the duration of the incubation period is short compared to commercial steels, being more similar to observations of high purity metals. This result is expected; our model material is precipitate- and solute-free. We also do not include the processes of dislocation loop formation and evolution, which would be expected to prolong the incubation. Finally, experimental observations of high-purity materials commonly show very little incubation delay, if any.

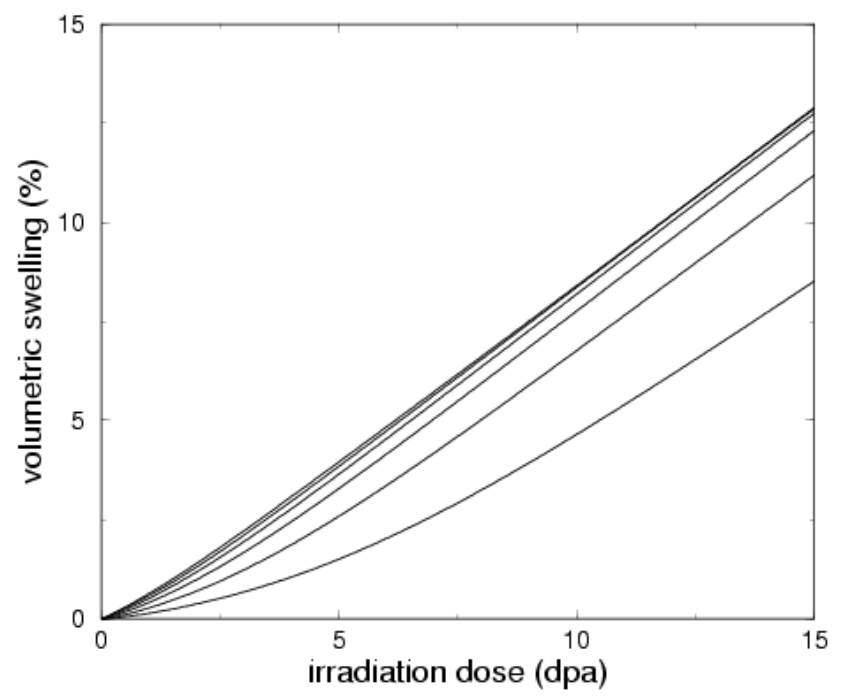

Fig.1 Volumetric swelling, $\Delta V / V$ in percent, versus total irradiation dose for a pure, type 316 stainless steel. The dose rate is $10^{-6} \mathrm{dpa} / \mathrm{s}$; the starting dislocation density is $6 \times 10^{13} \mathrm{~m}^{-2}$. The various curves correspond to temperatures of $340 \mathrm{C}$ to $540 \mathrm{C}$ in increments of $40 \mathrm{C}$. The $340 \mathrm{C}$ curve has the longest incubation period. While the dislocation density is allowed to evolve with time, the dislocation bias factors derived from the stress-induced interaction with the vacancy and interstitial defects is taken to be constant.

Our swelling predictions are very sensitive to the choice of dislocation bias factors. Fig. 2 displays the swelling curves using dislocation bias factors derived from earlier models (Sniegowski and Wolfer 1983). This is the same approach as used in Fig. 1, but whereas (Wehner and Wolfer 1985) calculate the bias factor at a fixed dislocation density near the asymptotic value, here the bias factors are calculated according to the instantaneous dislocation density. The predicted dislocation bias factors are found to be smaller at low dislocation densities, for example 1.39 and (1.03) for interstitials (vacancies) at a dislocation density of $6 \times 10^{13} \mathrm{~m}^{-2}$. This gives rise to a prolonged period of void nucleation in solution-annealed metals, producing a striking, incubation-like behavior that lasts from 2 to 15 dpa of total fluence. Besides lengthening the incubation period, the density-dependent bias also causes the asymptotic rates of swelling to depend 
noticeably on temperature. This change in behavior occurs because the terminal dislocation density is temperature-dependent, the calculated dislocation bias factors are now density-dependent, and the final swelling rates are strongly bias-dependent.

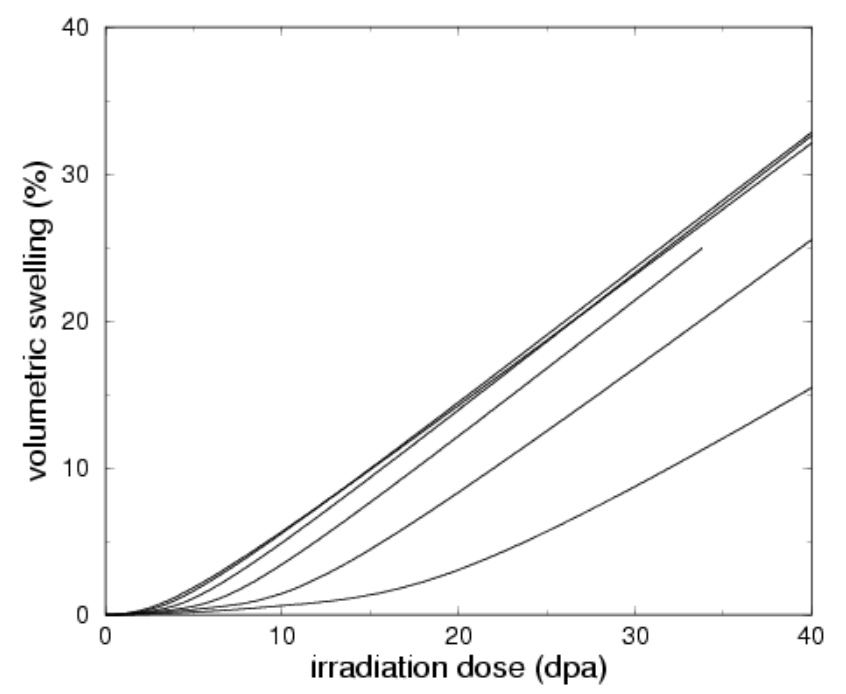

Fig.2 Volumetric swelling, $\Delta V / V$ in percent, versus total irradiation dose (equivalently, the irradiation time, in Msec) for a pure, type-316 stainless steel. The system parameters are exactly as in Fig. 1, except that, here, the dislocation bias factors are allowed to vary according to the momentary dislocation density of the model. Again, the lowest temperature curves display the longest incubation delays.

There is a striking difference in the predicted number density of voids between the constant and the variable dislocation-bias simulations, which can be seen in Figs. 3 and 4. The constant dislocation-bias approximation initially makes the dislocations stronger interstitial sinks. Thus, the simulation nucleates a large number of voids very quickly, accounting for the short incubation. There is also a small, transient overpopulation of small clusters. They become unstable and evaporate as the larger clusters grow, become stronger vacancy sinks, and reduce the vacancy supersaturation. At the end of this brief transient, void formation has been completed, nucleation of new, critical-size voids ceases, and the visible void number density is essentially constant. In contrast, the void population shows much more complex behavior in the time-dependent dislocation-bias simulation, with apparently multiple bursts of void nucleation followed by reductions (Fig. 3). The predicted terminal void population is several times smaller with the variable dislocation bias than in the constant dislocation-bias case (Fig. 4).

Comparing Fig. 2 to Fig. 3 shows that the incubation period is coincident with void nucleation. The steady-state swelling regime is achieved at essentially the same time that void nucleation is completed. 


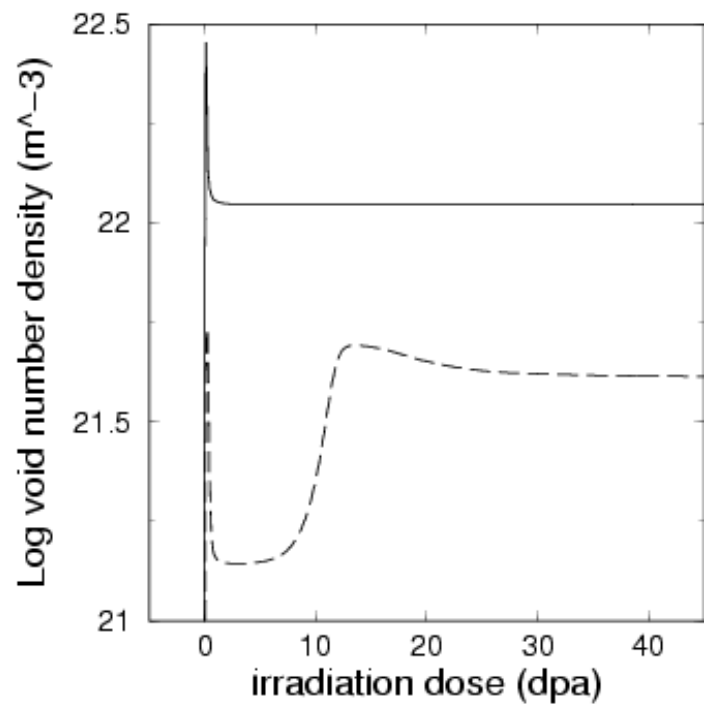

Fig. 3 Void number densities versus dose for type-316 stainless steel. These are "visible" voids, with radii of $0.5 \mathrm{~nm}$ or greater. Both curves correspond to irradiation at $10^{-6} \mathrm{dpa} / \mathrm{s}$ and $340 \mathrm{C}$. The solid curve is calculated with a fixed dislocation bias factor as used in Fig. 1. The dashed curve corresponds to the evolution with a variable dislocation bias, as in Fig. 2.

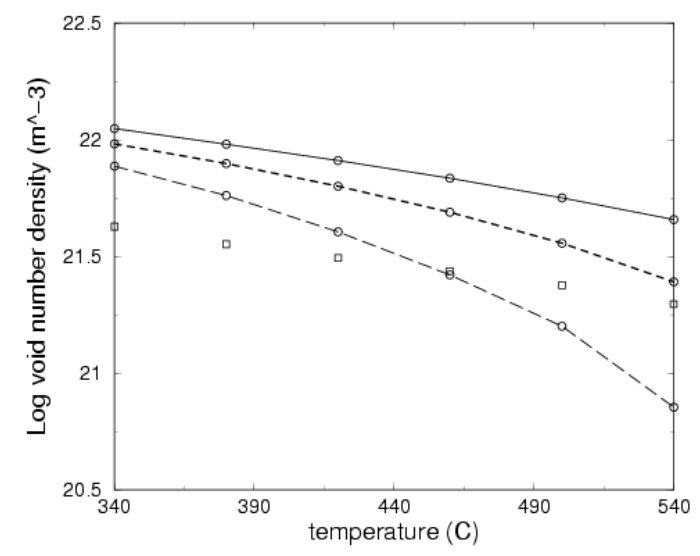

Fig. 4 Terminal void number density versus temperature and dose rate for type-316 stainless steel. All simulations are started at a dislocation density of $6 \times 10^{13} \mathrm{~m}^{-2}$. The data with lines to guide the eye are all obtained using the constant dislocation bias factors. The solid line is for a dose rate of $10^{-6} \mathrm{dpa} / \mathrm{s}$; the dashed line is $10^{-7} \mathrm{dpa} / \mathrm{s}$; and the long-dashed line is at $10^{-8} \mathrm{dpa} / \mathrm{s}$. The square points with no connecting line represent a calculation with the variable dislocation bias factors for a dose rate of $10^{-6} \mathrm{dpa} / \mathrm{s}$.

We can provide a quantitative estimate of the incubation period as the cumulative irradiation dose required to achieve a swelling of $1 \%$. This quantity is plotted versus temperature and dose rate in Fig. 5. We can see that the lower dose rates require a smaller incubation dose to complete the nucleation process. This is general agreement with the experimental observations of (Okita, et. al.) for high-purity ternary steels. The predicted incubation times are much shorter than are seen in conventional, commercial steels. Differences are expected, since the effect of impurities is not included in our 
model. Fig. 5 also demonstrates the sensitivity of incubation to the chosen dislocation bias factors. The use of a density-dependent dislocation bias factor greatly slows the rate of void nucleation, since the dislocation-interstitial bias is predicted to be smaller at low dislocation densities.

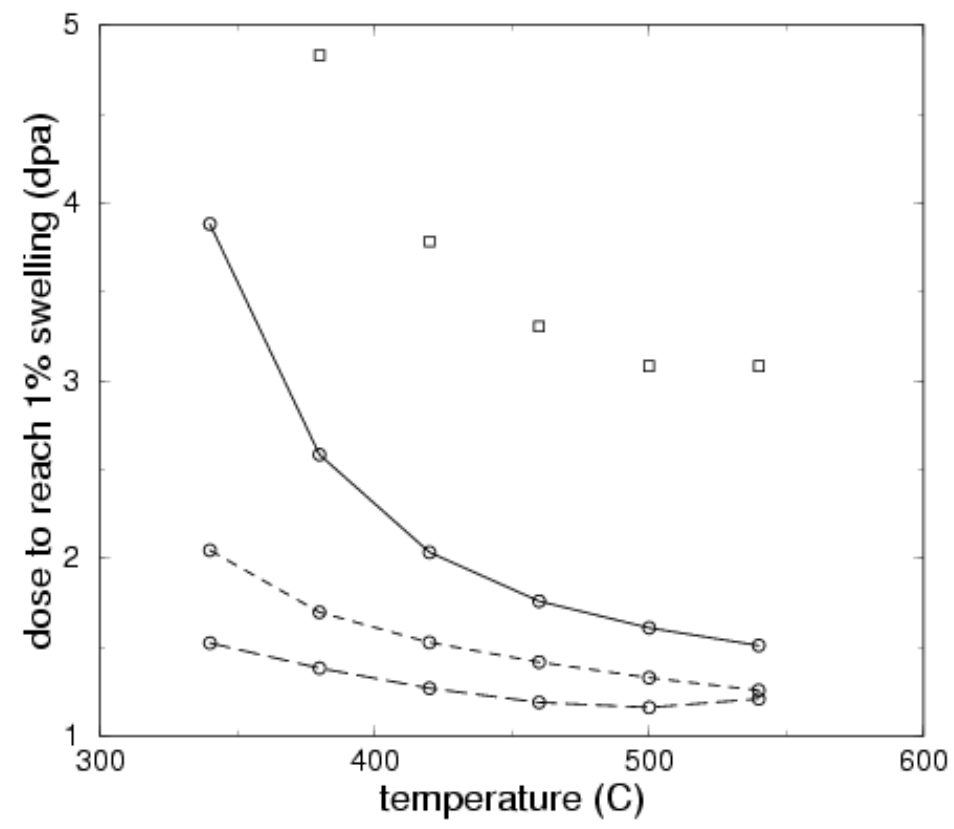

Fig. 5 Incubation times for the calculations with fixed and variable dislocation bias. The three curves are for calculations using the constant dislocation bias factors for dose rates of $10^{-6} \mathrm{dpa} / \mathrm{s}$ (solid line); $10^{-7} \mathrm{dpa} / \mathrm{s}$ (dashed line); and $10^{-8} \mathrm{dpa} / \mathrm{s}$ (long-dashed line). The squares are calculated using the variable dislocation bias factor, at $10^{-6} \mathrm{dpa} / \mathrm{s}$.

\section{Conclusion}

Generally speaking, the constant dislocation bias calculations are very close to the results of Wehner and Wolfer. This is despite differences in the numerical methods and the materials parameters, and although the dislocation density starts here at a different value $\left(6 \times 10^{13} \mathrm{~m}^{-2}\right.$ instead of $\left.2 \times 10^{13} \mathrm{~m}^{-2}\right)$ and is time-dependent, as well. For example, the terminal void number densities reported there are similar in magnitude to the results reported here. The results in Wehner and Wolfer differ mainly in exhibiting larger temperature dependence, in apparent better agreement with experiment. The use of a constant, larger-valued dislocation-interstitial bias reduces the predicted interstitial concentration, reduces vacancy-interstitial annihilation, increases the predicted vacancy supersaturation, and enhances void nucleation at early times. Stable voids have all formed within 0.1-0.2 dpa total fluence, before the dislocation density undergoes much change from its initial values. Thus, the results in Fig. 1 are expected to resemble Wehner and Wolfer where a time-independent dislocation density is used. The swelling in Fig. 2 with lower void number densities (square points in Fig. 4) develops because the variable dislocation bias factor for the interstitials is smaller for solution annealed material. The smaller bias leaves behind more interstitials to annihilate with vacancies 
and reduce their concentration. In turn, the reduced vacancy supersaturation reduces the void nucleation rate and prolongs the incubation period. In the subsequent steady-state regime, the variable dislocation-interstitial bias factors become roughly equal to the chosen constant values. The interstitial-vacancy segregation occurs at a roughly constant rate dictated by the dislocation bias, and the swelling rates in Figs. 1 and 2 are very similar. This is consistent with the observed, universal swelling rate versus temperature and irradiation dose rate.

To summarize, we report simulations of combined dislocation evolution with nucleation and growth of voids under irradiation. The simulations show a realistic swelling behavior for the coupled co-evolution. Because incubation of swelling and nucleation of voids appear to be coincident, a realistic theory of the incubation stage requires the treatment of void nucleation rates as well as systematic growth rates. This is achieved here by a treatment of stochastic cluster aggregation and evaporation.

While the dislocation time-evolution itself presents relatively simple, sigmoidal behavior, it can have dramatic effects on the void populations versus time if the dislocation bias factors depend on the density or dislocation arrangements (see Fig. 4). Notably, the bias factors may be quite different when the dislocations are gathered into tangles (Lethwaite 1982) or cell walls, thus the incubation behavior could also be influenced by dislocationdislocation spatial correlations. We are continuing to study this model, and plan to include production bias, dislocation loop formation and evolution, and solute impurities and gas in later versions.

\section{Acknowledgements}

This work was performed under the auspices of the U.S. Department of Energy by the University of California, Lawrence Livermore National Laboratory under Contract No. W-7405-Eng-48.

\section{References}

Brailsford, A.D. and Bullough, R., J. Nucl. Mater. 44121 (1972).

Garner, F.A., J. Nucl. Mater. 122, 459 (1984).

Garner, F.A., J. Nucl. Mater. 205, 98 (1993).

Katz, J.L. and Wiedersich, H., J. Chem. Phys. 55, 1414 (1971).

Lethwaite, G.W., Phil. Mag. A \{\bf 46\}, 653 (1982).

Okita, T, Sato, T., Sekimura, N., Garner, F.A., Greenwood, L.R., Wolfer, W.G., and Isobe, Y., to be published. 
Russell, K.C., Acta. Metal. 19, 753 (1971).

Sniegowski, J.J. and Wolfer, W.G., in Proceedings of Topical Conference on Ferritic Alloys for Use in Nuclear Energy Technologies, J.W. Davis and D.J. Michel, ed., (Snowbird, Utah, 1983), 579.

Surh, M.P., Sturgeon, J.B., and Wolfer, W.G., to be published.

Wehner, M.F. and Wolfer, W.G., Phil. Mag. A 52, 189 (1985).

Wolfer, W.G. and Glasgow, B.B., Acta. Metall. 33, 1997 (1985). 